\title{
Aplicação das classificações do sistema de informação estatística brasileiro à cadeia produtiva óleo-suco-citrícola nacional
}

\author{
Application of the Brazilian statistical information system classifications to the national citrus \\ oil-juice productive chain
}

\author{
Adailson da Silva Santos ${ }^{I}$ Leila Costa de Souza Santos ${ }^{I I}$
}

\section{RESUMO}

O trabalho aborda a aplicação das classificações do Sistema de Informação Estatística em vigência no Brasil na cadeia produtiva agroindustrial, preenchendo assim uma lacuna existente na literatura das ciências agrárias. Devido ao seu destaque no setor, em termos de produção/ comercialização/ exportação, selecionou-se a cadeia produtiva da indústria brasileira óleo-suco-citrícola. O objetivo é identificar o posicionamento de cada classificação na cadeia e as demandas a jusante e a montante para cada atividade e produto. Considerando o sistema estatístico, foi analisada a Classificação Nacional de Atividades Econômicas (CNAE) e a Nomenclatura Comum do Mercosul (NCM). Foram consultadas as informações estatísticas do Instituto Brasileiro de Geografia e Estatística (IBGE) e da Organização das Nações Unidas (ONU), além da específica literatura científica. De modo geral, essas classificações de atividades econômicas e produtos não são mutuamente excludentes, mas podem sobrepor-se umas às outras com relativa facilidade e mínima intervenção. Apesar das muitas segmentações existentes, tanto a cadeia produtiva, quanto as classificações deste trabalho encontram-se corretamente definidas. Os elos da cadeia produtiva da indústria óleo-suco-citrícola brasileira envolvem estas atividades: cultivo, produção, colheita, processamento dos sucos e afins, aproveitamento industrial de subprodutos e resíduos/refugos, packing houses, distribuição, comercialização e consumo. Os produtos são: insumos agrícolas, a laranja (fruta), os sucos, óleos essenciais, águas aromáticas, terpenos, terpenoides, limoneno, perfumes e cosméticos.

Palavras-chave: sistema de informação estatística, classificação, CNAE, NCM, cadeia produtiva, laranja, óleo essencial.

\begin{abstract}
This paper addresses the application of the Statistical Information System classifications in force in Brazil in the agro-industrial production chain, thus filling a gap in the literature of agricultural sciences. Due to its prominence in the sector, in terms of production / marketing / exports, it was selected the productive chain of the oil-juice-citrus Brazilian industry. The goal is to identify each classification position in the chain and downstream and upstream demands for each activity and product. Considering the statistical system, it was analyzed the National Classification of Economic Activities (CNAE) and the Mercosul Common Nomenclature (NCM). It was consulted the statistical information of the Brazilian Institute of Geography and Statistics (IBGE) and the United Nations $(U N)$, besides the specific scientific literature. In general, these classifications of economic activities and products are not mutually exclusionary but can overlap each other with relative ease and minimal intervention. Despite the many existing segmentation, both the supply chain and the classifications from this work are set correctly. The productive chain of the oiljuice-citrus Brazilian industry involves these activities: cultivation, production, harvesting, processing of juices and related, industrial utilization of by-products and waste / scrap, packing houses, distribution, marketing and consumption. The products are: agricultural inputs, orange (fruit), juices, essential oils, aromatic waters, terpenes, terpenoids, limonene, fragrance and cosmetics.
\end{abstract}

Key words: Statistical information system, Classification, CNAE, NCM, Productive Chain, Orange, Essential oil.

'Divisão de Petróleo e Engenharia Química, Diretoria de Patentes, Instituto Nacional da Propriedade Industrial (DIPEQ/DIRPA/ INPI), 20090-910, Rio de Janeiro, RJ, Brasil. E-mail: adailson@inpi.gov.br. Autor para correspondência.

"Coordenação Geral Regional Rio de Janeiro, Divisão de Inovação e Prospecção Tecnológica, Seção de Propriedade Intelectual e Inovação, Instituto Nacional de Tecnologia (CGRRJ/DINT/SPIN/INT), Rio de Janeiro, RJ, Brasil. 


\section{INTRODUÇÃO}

A citricultura caracteriza-se por ser um dos setores mais competitivos do agronegócio nacional, com o Brasil detendo 30\% da produção mundial da fruta laranja e mais de $60 \%$ da produção de suco de laranja. O sistema agroindustrial citrícola brasileiro movimenta $\mathrm{R} \$ 9$ bilhões por ano com as exportações de suco de laranja, alcançando US\$ 1,2 bilhão (aproximadamente, 60\% das exportações vão para a União Europeia e 15\% para os Estados Unidos), além da geração de mais de 400 mil empregos diretos e indiretos em todo o setor agrário (NEVES et al., 2006).

O parque produtor da citricultura paulista é o mais importante em todo o país, dominando, junto com o estado norte-americano da Flórida, a oferta mundial. A atividade exportadora não se restringe à laranja e seu suco, mas, ainda, às exportações do farelo de polpa cítrica e dos óleos essenciais cítricos (particularmente, o da laranja) (SILVA-SANTOS, 2002). Além da indústria alimentícia, essa cadeia produtiva atende a diferentes segmentos de consumidores industriais, como cosmética, perfumaria e farmacêutica (fornecendo óleos essenciais, seus constituintes e derivados, e a pectina cítrica).

Ao longo dos últimos vinte anos, o óleo essencial de laranja respondeu por $80 \%$ das exportações atuais do segmento das indústrias produtoras de óleos essenciais, sendo o primeiro item na pauta nacional de produção, comercialização e exportação desse setor (SILVA-SANTOS, 2002).

O presente trabalho aborda a aplicação prática das classificações existentes no Sistema de Informação Estatística em vigência no Brasil na cadeia produtiva agroindustrial, abordando o tema das taxonomias das atividades econômicas e de produtos. Tema este que pesquisadores e empresários do setor, sistematicamente se deparam em seus levantamentos de dados e análises, particularmente, em referência às questões tributárias e de balança comercial. Contudo, por não serem profissionais do setor ou não terem a disponibilidade de tempo para o conhecimento mais profundo, são rotineiras as ocorrências de problemas no uso de tais classificações e sistemas, levando à reclassificação (duplicação de tempo/trabalho) ou ao indeferimento (negação) dos documentos tributários ou solicitações apresentados aos órgãos da administração nacional (pedido de bolsas/apoio financeiro etc.). Assim, justamente por isso, há a necessidade de preencher tal lacuna existente na literatura das ciências, neste caso particular, a agrária.

Para tanto, foi realizado um exercício de natureza prática, em que foram aplicadas à cadeia produtiva da indústria brasileira óleo-suco-citrícola as classificações existentes no Sistema de Informação Estatística brasileiro (visto ser uma das que mais se destacam no setor, em termos de produção/ comercialização/ exportação), de forma pontual, identificando-se quais códigos taxionômicos referemse às atividades de produção agropecuária e industrial e aos produtos ali obtidos. O objetivo é identificar como cada classificação se posiciona na cadeia produtiva e compreender como elas se inter- e/ou intrarrelacionam entre si, para cada atividade e produto nela indicados. Consideraram-se, ainda, no seu caráter mais amplo (lato sensu) possível, os atores mais externos a esta cadeia (por exemplo, os principais clientes), como as indústrias alimentícias, cosméticas e de aromas/fragrâncias (todos como fabricantes de produtos de uso final). A seguir, apresenta-se a teoria econômica sobre os conceitos de cadeia produtiva e sistema de classificação necessários para a realização deste trabalho.

A cadeia produtiva, ou "cadeia de valor", consiste no arranjo das atividades necessárias para produzir um bem ou serviço, desde a sua concepção, passando pelas diferentes fases da produção até a entrega ao consumidor final (SOUZA \& AMATO NETO, 2005; BATALHA, 1997; BATALHA, 1995). Esse conceito originou-se no setor agrícola, a partir da ampliação da visão das atividades realizadas dentro das fazendas para fora delas mesmas, a jusante ou a montante. Com isso, foi criado o conceito de agribusiness (também denominado de "complexo agroindustrial", "negócio agrícola" ou "agronegócio") definindo não apenas o que ocorria dentro dos limites das propriedades rurais, mas todos os processos interligados que propiciam a oferta dos produtos aos seus consumidores (ZYLBERSZTAJN, 1994). Nessa concepção, uma cadeia produtiva agropecuária seria composta por elos que englobariam as organizações supridoras de insumos básicos para a produção agrícola ou agroindustrial, as fazendas e agroindústrias com seus processos produtivos, as unidades de comercialização atacadista e varejista e os consumidores finais, todo conectados por fluxos de capital, materiais e de informação (NEVES et al., 2001).

$\mathrm{O}$ conceito extrapolou para outras áreas produtivas, tornando-se universal e permitindo utilizar as suas capacidades e ferramentas analíticas para a formulação de estratégias e políticas de desenvolvimento em uma ampla gama de processos produtivos (CASTRO et al., 2002).

Ao longo das décadas de 1980 e 1990, várias contribuições ampliaram o uso do enfoque sistêmico e de cadeias produtivas em estudos e projetos de desenvolvimento, melhorando a compreensão, 
intervenção e a gestão no desempenho da atividade industrial, como, por exemplo, o estudo realizado por CAMILLE VERT (1991), do qual deriva o modelo geral de cadeia produtiva adotado neste trabalho (SILVASANTOS, 2002). Esse modelo consiste em uma sequência de elos ou blocos que englobam as principais categorias de atividades industriais praticadas: produtoras de matérias-primas (ou produtora de insumos); transformadoras (ou formuladoras); embaladoras; utilizadoras finais e traders. O Sistema de Classificação Econômica integra o chamado Sistema de Informação Estatística (SIE) e consiste no conjunto de informações estatísticas oficiais coletadas, tratadas, organizadas, analisadas e disseminadas por um órgão estatístico central, visando ao conhecimento da realidade econômica e social do país. Todos os processos econômicos que devem ser descritos mediante estatísticas requerem uma classificação sistemática (PORCARO, 2000). As instituições responsáveis pela manutenção, atualização e revisão das classificações são denominadas órgãos estatísticos centrais ou gestores de classificações. Cada gestor fica com a responsabilidade de conduzir a manutenção, atualização e revisão de sua(s) própria(s) classificação(ões). No caso brasileiro, o órgão estatístico central é o Instituto Brasileiro de Geografia e Estatística (IBGE), criado por legislação oficial específica e definido como órgão coordenador e principal produtor do Sistema de Informação Estatística Nacional (SEN).

Dentre as classificações existentes, neste trabalho, foi dada particular atenção às classificações de atividades econômicas e de produtos. As classificações de atividades econômicas conectam-se ao conjunto de agentes do processo de produção, estando distribuídas em diferentes modalidades: produtivas, ou seja, de bens e serviços de manutenção/ reparação de máquinas e equipamentos e de apoio; atividades diretamente associadas ao processo produtivo, como produção de utilidades, prestação de serviços de controle de qualidade, desenvolvimento de projetos e tratamento de poluentes; e atividades indiretamente associadas à produção, como as administrativas e auxiliares.

As classificações de produtos (incluindo os químicos), integrantes do conjunto dos bens e serviços, promovem o agrupamento dos dados segundo as características físicas, tipos de insumo, etapas do processo produtivo, origem no processo produtivo e mercado ou finalidade de uso. As estatísticas elaboradas com essa classificação abrangem a produção, consumo final e intermediário (também chamado de "consumo industrial", ou seja, o que é consumido pelas indústrias para gerar o produto final), análise dos fluxos de bens e serviços na economia, formação de capital, comércio exterior ou preços (BORSCHIVER et al., 2004).

A "Família Internacional de Classificações Econômicas e Sociais" criada pela Organização das Nações Unidas (ONU) é a utilizada atualmente pela maioria dos países como padrão de referência para as classificações de atividades econômicas e produtos, dentre outras. Essa Família engloba três principais níveis hierárquicos (BORSCHIVER et al., 2004):

a) Classificações de referência - resultantes dos acordos internacionais aprovados pela United Nation Statistical Commission (ou outro órgão intergovernamental competente, dependendo do tema envolvido). Essas classificações são de ampla aceitação e concordância oficial, sendo aprovadas e recomendadas como referências (guidelines) para o desenvolvimento de outras classificações, tanto em relação à estrutura quanto em relação ao caráter e definição das categorias;

b) Classificações derivadas - baseadas nas classificações de referência e preparadas tanto pela (a) adoção das categorias e da estrutura das classificações de referência, às vezes incluindo um detalhamento adicional àquele fornecido pela classificação de referência, ou (b) por meio da reorganização ou agregação de itens de uma ou mais classificações de referência;

c) Classificações relacionadas - referem-se parcialmente às classificações de referência, ou são associadas a estas apenas em alguns níveis específicos de sua estrutura.

Devido aos diferentes níveis hierárquicos das classificações integrantes da "Família Internacional", as informações colhidas são processadas em um grau de detalhamento maior ou menor, refletindo na adoção de níveis de codificação hierárquica mais ou menos extensos, que podem conter uma quantidade maior ou menor de dígitos para cada código adotado.

Dentre a "Família Internacional", destacamse as classificações de atividade e de produtos mais amplamente empregadas nos SIE dos países europeus e das Américas, em cada um dos três principais níveis hierárquicos existentes (referências, derivadas e relacionadas), como apresentado na tabela 1 .

As duas principais classificações de atividades econômicas e de produtos empregados no 
Tabela 1 - Principais classificações de atividades econômicas e de produtos e gestores.

\begin{tabular}{|c|c|c|c|}
\hline Tipo & Nomenclatura & Hierarquia & Gestor \\
\hline \multirow{3}{*}{ Atividade econômica } & $\operatorname{ISIC}^{a}$ & Referência & $\mathrm{UNSD}^{e}$ \\
\hline & $\mathrm{NACE}^{b}$ & Derivada & SEC/E.U. ${ }^{f}$ \\
\hline & $\begin{array}{l}\text { NAICS-USA }^{c} ; \\
\text { CNAE }^{d}\end{array}$ & Relacionada & $\begin{array}{l}{\mathrm{ECPC} / \mathrm{USA}^{g}}^{g} ; \\
\mathrm{IBGE}^{h}\end{array}$ \\
\hline \multirow{3}{*}{ Produto } & $\begin{array}{l}\mathrm{CPC}^{i} \\
\mathrm{SH}^{j}\end{array}$ & Referência & $\begin{array}{l}\mathrm{UNSD}^{e} ; \\
\mathrm{WCO}^{m}\end{array}$ \\
\hline & $\operatorname{SITC}^{k}$ & Derivada & $\mathrm{UNSD}^{e}$ \\
\hline & $\mathrm{NCM}^{l}$ & Relacionada & Mercosul $^{n} / \mathrm{IBGE}^{h}$ \\
\hline
\end{tabular}

FONTE: Adaptado pelo autor de UNIÃO EUROPÉIA (2007a).

Obs.: a ISIC - International Standard Industrial Classification of All Economic Activities; $b$ NACE - Nomenclature Générale des Activités Économiques dans les Communautés Européennes; c NAICS-USA - North American Industry Classification System of the United States of America; $d$ CNAE - Classificação Nacional de Atividades Econômicas (Brasil); e UNSD - United Nation Economic Statistics and Classifications Section; $f$ SEC/E.U. - Statistical Office of the European Community; $g$ ECPC/OMB - Economic Classification Policy Committee (ECPC) of United States of America; $h$ IBGE - Instituto Brasileiro de Geografia e Estatística; $i$ CPC - Central Product Classification; $j \mathrm{SH}$ - Harmonized System; ${ }^{k}$ SITC - Standard International Trade Classification; ${ }^{l, n}$ NCM - Nomenclatura Comum do Mercado Comum do Sul (MERCOSUL); $m$ WCO - World Customs Organization.

Sistema de Informação Estatística (SIE) brasileiro para as atividades econômicas e produtos são: (1) CNAE, Classificação Nacional de Atividades Econômicas, para as atividades econômicas; e (2) NCM, Nomenclatura Comum do Mercosul, para os produtos.

A Classificação Nacional de Atividades Econômicas (CNAE) foi criada em 1994 e é gerenciada pelo Instituto Brasileiro de Geografia e Estatística (IBGE). A CNAE é o instrumento de padronização utilizado pelos diversos órgãos da Administração Tributária do Brasil, como a Receita Federal. A partir de acordos internacionais assinados pelo Brasil, foi adotada uma estrutura padronizada baseada na ISIC (International Standard Industrial Classification of All Economic Activities), tornando-se uma classificação-relacionada a ela mesma (CONCLA, 2007; SILVA, 2005). Apesar de ser uma classificaçãorelacionada a ISIC, sua estrutura difere desta, empregando um sistema numérico de até sete dígitos, enquanto a ISIC utiliza um sistema exclusivo de quatro dígitos. Atualmente, encontra-se em vigor a versão CNAE 2.0.

A Nomenclatura Comum do Mercosul (NCM) para a classificação de produtos originou-se a partir das decisões tomadas em 1986, quando o CCC (Customs Cooperation Council), em conjunto com a UNSD (United Nation Economic Statistics and Classifications Section) e outros órgãos estatísticos nacionais e internacionais, aprovou o Harmonized Commodity Description and Coding System ou Harmonized System (HS), uma nomenclatura comum de referência para atender aos interesses aduaneiros e estatísticos envolvidos no comércio internacional (BORSCHIVER et al., 2004). O Sistema HS é uma classificação de produtos de referência que tem como órgão internacional responsável o World Customs Organization (WCO), e compreende um total de 7.466 grupos de produtos identificados, constituindo uma organização sistemática e uniforme de produtos.

No âmbito do MERCOSUL (Mercado Comum do Sul), é amplamente utilizada a Nomenclatura Comum do Mercosul (NCM) como classificaçãorelacionada à HS. A sistemática de classificação dos códigos na Nomenclatura Comum do Mercosul (NCM) obedece rigidamente à estrutura hierárquica da $\mathrm{HS}$, sendo acrescida, ao final, de dois dígitos. Dessa maneira, comparada à HS, a NCM contém um nível a mais (Subcategorias), e a codificação é composta, no total, por 8 dígitos (SILVA-SANTOS, 2002).

\section{DESENVOLVIMENTO}

Aplicou-se a fundamentação econômicoteórica acerca (1) das cadeias produtivas e (2) das classificações industriais de atividades econômicas e de produtos à cadeia produtiva óleo-suco-citrícola da laranja, em cada uma das etapas produtivas integrantes, bem como aos produtos relacionados.

Foi utilizado o modelo geral de cadeia produtiva de CAMILLE VERT(1991), em que, para cada atividade econômica realizada e produto obtido, será inferido o respectivo código taxionômico. Para tanto, selecionaram-se, na "Família Internacional de Classificações Econômicas e Sociais”, regidas pela 
Organização das Nações Unidas (ONU), as classificações utilizadas no Sistema de Informação Estatística brasileiro, isto é, CNAE (Classificação Nacional de Atividades Econômicas) e NCM (Nomenclatura Comum do Mercosul) em suas versões mais recentes.

Observa-se que os elos da cadeia produtiva óleo-suco-citrícola brasileira aqui considerados, segundo NEVES et al. (2001), envolvem os insumos agrícolas, cultivo e produção, colheita, indústria processadora dos sucos e afins, aproveitamento industrial de subprodutos e resíduos/refugos, packing houses, distribuição, comercialização e consumo. Neste último, considerou-se, ainda, no seu caráter mais amplo (lato sensu) possível, os atores mais externos a esta cadeia, como as indústrias alimentícias, cosméticas e de aromas/ fragrâncias (principais clientes e fabricantes de produtos de uso final).

A partir das informações disponibilizadas e reordenadas através do modelo de $\mathrm{C}$. Vert, verificou-se como a cadeia produtiva óleo-suco-citrícola nacional pode ser representada através da taxionomia das classificações de atividades econômicas (Classificação Nacional de Atividades Econômicas - CNAE) e de produtos (Nomenclatura Comum do Mercosul - NCM) presentes no Sistema de Informação Estatística Brasileiro. Os resultados da taxonomia para o caso da cadeia produtiva óleo-suco-citrícola da laranja são apresentados na figura 1 .

Os elos representados da cadeia da laranja envolvem os insumos, cultivo e produção, colheita, indústria processadora dos sucos e afins, aproveitamento industrial de subprodutos e resíduos/ refugos, packing houses, distribuição, comercialização e consumo. Durante o beneficiamento dos óleos essenciais cítricos, a aplicação de outros processos industriais permite a obtenção de outros produtos, por exemplo, o isolamento de componentes terpênicos ou terpênico-oxigenados (também chamados terpenoides). Cabe ressaltar que o isolamento destes compostos terpênico-oxigenados não integra o capítulo CNAE 2029-1/00 (representado na Figura 1), estando inserido em posição aqui não abordada (por não ser o foco do trabalho).

Da mesma forma, a subcadeia produtiva das águas aromáticas residuais (NCM 3301.90.30) não foi abordada na figura 1, já que o foco do trabalho é a parte "óleo-suco" da cadeia principal.

Outra observação é que tanto as águas aromáticas residuais quanto o limoneno (um composto terpênico), ambos representados na figura 1, também são importantes e utilizados no capítulo CNAE 2063-1/ 00, para a produção de produtos das posições NCM
3303, 3304 ou 3307. Outras indústrias não representadas na figura 1 também utilizam intensamente as águas aromáticas residuais, compostos terpênicos, compostos terpênico-oxigenados e os óleos essenciais, por exemplo, indústria farmacêutica, biocidas, domissanitária, higiene pessoal, etc. Em relação à própria fruta laranja, apesar de não ser apresentada na figura 1, ela possui um código CNAE específico para a sua comercialização (CNAE 4633-8/01).

A finalidade principal das classificações aqui empregadas é a de promover a taxionomia das atividades e produtos na sua forma mais detalhada e específica. Contudo, este detalhamento também acaba gerando inúmeras categorias e subcategorias, nas quais pesquisadores e empresários envolvidos com o tema ou que necessitam usar tais ferramentas, podem defrontar-se com erros de taxionomia e de análise.

\section{CONCLUSÃO}

Percebeu-se a variedade de classificações às quais as atividades e produtos são subordinados. De modo geral, estas não são excludentes entre si, mas podem sobrepor-se umas às outras com relativa facilidade e mínima intervenção.

Os procedimentos de manutenção, atualização e revisão das classificações estatísticas da Família Internacional visam à resolução de problemas de correspondência e fornecem a oportunidade de aumentar a harmonização daquelas classificações.

As nomenclaturas derivadas, de uma forma geral, são aquelas que apresentam a maior quantidade de itemizações, por terem um maior número de níveis e de dígitos empregados.

Verificou-se o necessário estudo aprofundado de toda a cadeia produtiva a ser considerada, identificando-se os elos e os demais elementos que a compõem. Dessa maneira, é possível aplicar corretamente as classificações a cada tópico particular da cadeia. Outra consideraçãoé a necessidade de compreender o detalhamento existente em cada sistema de classificação, evitando-se a ocorrência de erros de taxionomia e de análise.

O correto conhecimento dos códigos a serem aplicados para as atividades e produtos, destrava ou agiliza procedimentos burocráticos referentes às questões envolvendo Receita Federal e práticas de Comércio Exterior, assegurando o adequado levantamento de dados e análises que, por exemplo, pesquisadores e empresários se deparam rotineiramente, particularmente, no tocante às questões tributárias e de balança comercial.

A cadeia produtiva considerada neste trabalho está bem definida, apesar das muitas 


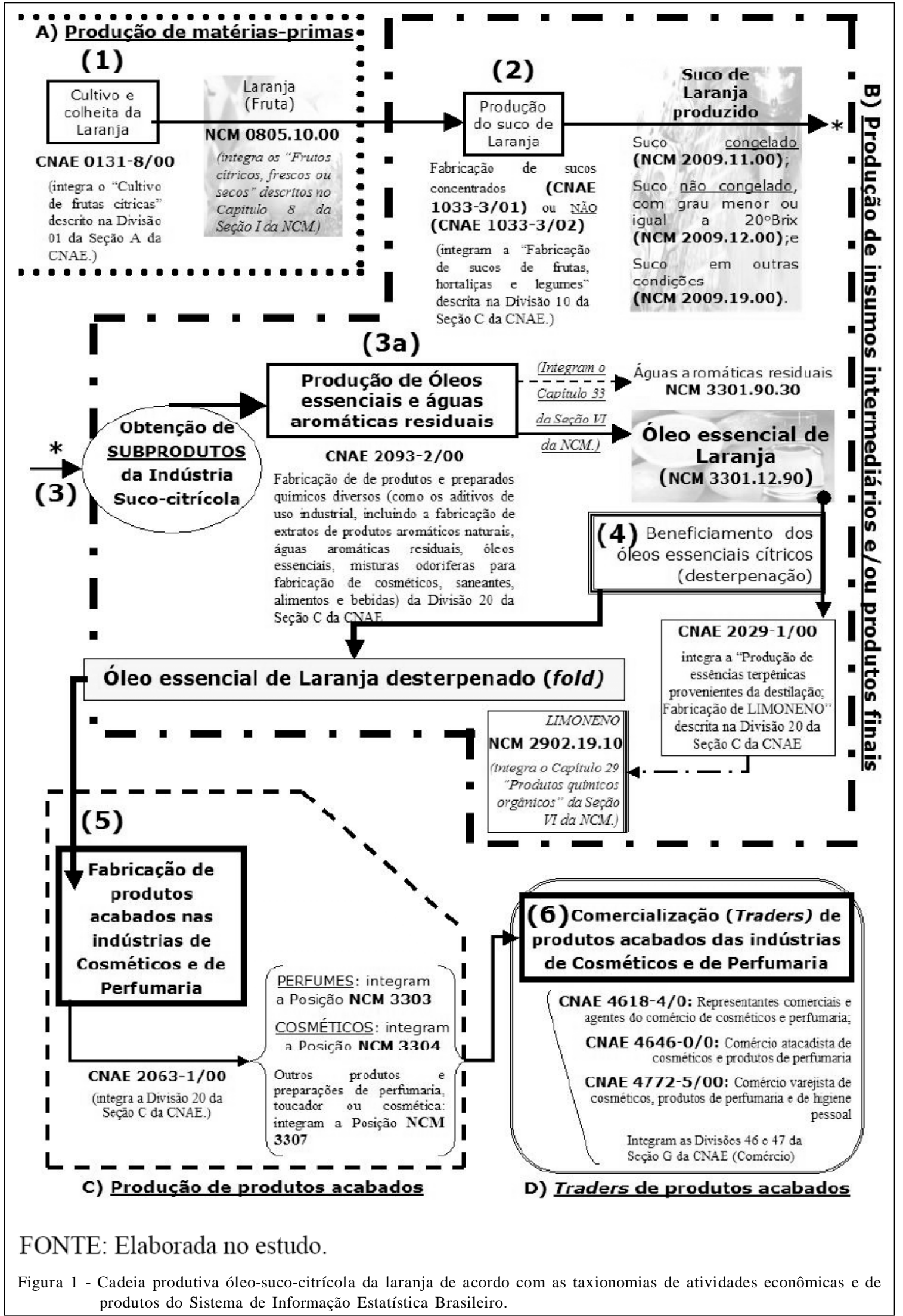

Ciência Rural, v.41, n.4, abr, 2011. 
segmentações existentes. Similarmente, as classificações de produtos e atividades também estão corretamente definidas, facilitando sua aplicação pontual.

\section{REFERÊNCIAS}

BATALHA, M.O. Sistemas agroindustriais: definições e correntes metodológicas. In: BATALHA, et al. Gestão agroindustrial. São Paulo: Atlas, 1997. V.1, p.24-48.

As cadeias de produção agro-industriais: uma perspectiva para o estudo das inovações tecnológicas. Revista de Administração da Universidade de São Paulo, São Paulo, v.30, n.42, p.43-50, 1995. Disponível em: <http:// www.rausp.usp.br/download.asp?file $=3004043$.pdf $>$. Acesso em: 07 fev. 2010

BORSCHIVER, S. et al. A classificação industrial e sua importância na análise setorial. Ciência da Informação, Brasília, v.33, n.1, p.9-21, 2004. Disponível em: <http:// www.scielo.br/scielo.php?script $=$ sci_arttext\&pid $=S 0100$ $19652004000100002 \& \operatorname{lng}=$ pt\&nrm=iso $>$. Acesso em: 12 maio, 2009. doi: 10.1590/S0100-19652004000100002.

CASTRO, A.M.G. et al. Cadeia produtiva: marco conceitual para apoiar a prospecção tecnológica. In: SIMPÓSIO DE GESTÃO DA INOVAÇÃO TECNOLÓGICA, 22., 2002, Salvador. Artigo... Salvador: PGT/USP, 2002. Acesso em: 21 abr. 2009. Online. Disponível em: <http://www.mdic.gov.br/ arquivos/dwnl_1197031881.pdf>.

COMISSÃO NACIONAL DE CLASSIFICAÇÃO (CONCLA). Subcomissão Técnica da CNAE-Fiscal: Estrutura. Acesso em: 07 ago. 2007. Online. Disponível em: <http:// www.cnae.ibge.gov.br/>.

MERCADO COMUM DO SUL (MERCOSUL). MERCOSUL/ GMC/RES. $\mathbf{N}^{\circ}$ 70/06. Tarifa externa comum incorporação à NCM da IV emenda ao sistema harmonizado de designação e codificação de mercadorias. Acesso em: 19 dez. 2008. Online. Disponível em: <http://www.mercosur.int/ $\mathrm{msweb} / \mathrm{S} \mathrm{M} / \mathrm{Actas} \% 20 \mathrm{~T}$ EMPORARIAS/GMC/ LXVI\%20 G MC \% 20 ACTA \% 2005-06\%20FINAL/

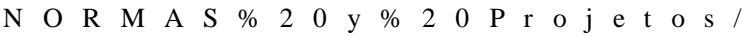
NORMAS $\% 20$ APROB ADAS $\% 20$ LXVI\% $20 \mathrm{GMC} /$ RESOLU\%C3\% $87 \%$ C $3 \% 95$ ES/GMC_Res_\% 2070 06\%20PT\%20-\%20IV\%20Enmienda.pdf>.

NEVES, M.F.; JANK, M.S. (Coords.). Perspectivas da cadeia produtiva da laranja no Brasil: A Agenda 2015. São Paulo, SP, 23/11/2006. Acesso em: 19 mar. 2009. Online. Disponível em: <http://www.fundace.org.br/arquivos_diversos/agenda_estrategica/ Agenda_Citrus_2015_PENSAICONE.pdf $>$.

NEVES, E.M. et al. Brazilian citrus industry: economic and financial effects, 1996-2000. Revista Brasileira de Fruticultura, Jaboticabal, v.23, n.2, 2001, p.432-436. Disponível em: <http:// www.scielo.br/scielo.php?script $=$ sci_arttext\&pid $=$ S0100$29452001000200048 \& \operatorname{lng}=e s \& n r m=i s o>$. Acesso em: 26 jun. 2009. doi: $10.1590 / \mathrm{S} 0100-29452001000200048$.

PORCARO, R.M. Produção de informação estatística oficial na (des)ordem social da modernidade. 2000. $194 \mathrm{f}$. p.21-25, p.34-35, p.73-74. Tese (Doutorado em Ciência da Informação) - Escola de Comunicação da UFRJ, Rio de Janeiro, RJ. Acesso em: 10 ago. 2009. Online. Disponível em: <http:/ /tede-dep.ibict.br/tde_busca/arquivo.php?codArquivo=23>.

SILVA, C.R.L. Contas regionais do Brasil, segundo a classificação nacional das atividades econômicas do período 1998 a 2001. Informações Econômicas, São Paulo, v.35, n.2, p.01-02, 2005. Disponível em: <ftp://ftp.sp.gov.br/ftpiea/seto2-0205.zip>. Acesso em: 03 maio, 2009

SILVA-SAnTOS, A. Análise técnica, econômica e de tendências da indústria brasileira de óleos essenciais. Rio de Janeiro: Papel Virtual, 2002. 202p.

SOUZA, R.C.; AMATO NETO, J. Cadeias de valor global: condições para a inserção de uma cooperativa exportadora de suco de maracujá concentrado no mercado global. Agricultura em São Paulo, São Paulo, v.52, n.2, p.5-15, 2005. Disponível em: <ftp://ftp.sp.gov.br/ftpiea/publicacoes/asp1-2-05.pdf>. Acesso em: 15 set. 2009.

UNIÃO EUROPEIA. General description of classification item 70, NACE Rev 2. Online. Disponível em: <http:// ec.europa.eu/eurostat/ramon/nomenclatures/ index.cfm?TargetUrl=LST_NOM\&StrLanguageCode=EN\&IntFami lyCode $=\&$ TxtSearch $=\& I n t C u r r e n t P a g e=2>$. Acesso em: $14 \mathrm{fev}$. 2009.

General description of classification item 39, HS 2002. Acesso em: 19 mar. 2009. Online. Disponível em: $<$ http://ec.europa.eu/eurostat/ramon/nomenclatures/ index.cfm?TargetUrl=LST_NOM\&StrLanguageCode=EN\&IntFam ilyCode $=\&$ T xtSearch $=\&$ IntCurrentPage $=1>$.

VERT, C. Marketing integrado. Lisboa: Monitor, 1991. $180 \mathrm{p}$.

ZYLBERSZTAJN. D. Agribusiness: Conceito, Dimensões e Tendências. Estudos de Política Agrícola, v. 28, p. 351-379, 1994. 